\title{
Safety implications of cultural and cognitive issues in nuclear power plant operation
}

\author{
Paulo V.R. Carvalho ${ }^{\mathrm{a}, *}$, Isaac L. dos Santos ${ }^{\mathrm{a}}$, Mario C.R. Vidal ${ }^{\mathrm{b}}$ \\ ${ }^{a}$ Comissão Nacional de Energia Nuclear, Instituto de Engenharia Nuclear Cidade Univerisitária, Ilha do Fundão, Rio de Janeiro, \\ CEP 21945-970, RJ, Brazil \\ ${ }^{\mathrm{b}}$ Grupo de Ergonomia e Novas Tecnologias, GENTE, COPPE/UFRJ, Cidade Universitária, Ilha do Fundão, Rio de Janeiro, RJ, Brazil
}

Received 11 November 2003; accepted 18 March 2005

\begin{abstract}
This research project was designed to investigate cultural and cognitive issues related to the work of nuclear power plant operators during their time on the job in the control room and during simulator training (emergency situations), in order to show how these issues impact on plant safety. The modeling of the operators work deals with the use of operational procedures, the constant changes in the focus of attention and the dynamics of the conflicting activities. The paper focuses on the relationships between the courses of action of the different operators and the constraints imposed by their working environment. It shows that the safety implications of the control room operators' cognitive and cultural issues go far beyond the formal organizational constructs usually implied. Our findings indicate that the competence required for the operators are concerned with developing the possibility of constructing situation awareness, managing conflicts, gaps and time problems created by ongoing task procedures, and dealing with distractions, developing skills for collaborative work.
\end{abstract}

(C) 2005 Elsevier Ltd. All rights reserved.

Keywords: Situated action; Nuclear power plant operation; Cognitive strategies

\section{Background}

As Wilson (2000) has shown, the study of Ergonomics has evolved around the world as one of the keys to understanding human behavior in interaction with socio-technical systems. The Nuclear Engineering Institute and the Ergonomics and New Technologies Group (GENTE) at the Federal University of Rio de Janeiro has initiated a research project to determine how ergonomics can be used in complex socio-technical systems (in nuclear, petroleum and aviation operations) to help prevent accidents (Carvalho and Vidal, 2001, 2003). This continuing research project uses both a practical and theoretical ergonomic approach to assess the cognitive strategies in the operations of nuclear

\footnotetext{
${ }^{*}$ Corresponding author. Tel.: + 552122098185.

E-mail address: paulov@ien.gov.br (P.V.R. Carvalho).
}

power plant (NPP) technicians, and more specifically how these technicians deal with formal organizational constructs (procedures, rules, norms) in their daily operations.

\section{Introduction}

The nuclear industry has long demonstrated its desire to avoid accidents if possible, and mitigate the consequences if not, by dealing with safety issues according to classical system safety engineering paradigms. Thus safety begins in the conceptual design stage and continues throughout the project life-cycle: in the design, production, testing, licensing, operational use, and decommissioning phases. The primary emphasis is on the early identification and classification of hazards so that action can be taken to eliminate or minimize 
these hazards before final design decisions are made. From the time of the first NPPs, hardware was based on defense-in-depth concepts, barriers, redundancies, diversity, high quality components, and so on. The nuclear industry also developed a sophisticated risk management system based on analytical safety control, considering postulated accident scenarios predicted from processes models, in order to develop probabilistic risk analysis (PRA) to estimate the probability of serious accidents.

Prior to the accident at Three Mile Island (TMI) in 1979, human factors received little attention in nuclear safety (Mosey, 1990). After TMI, all nuclear accidents have been reviewed, from Windscale to Chernobyl, and root cause analysis has shown "human error" to be a major component in all nuclear accidents. As a result, human error and human behavior have come to be treated in the same way as technical (hardware) systems. In this framework, the natural variability of complex human agents must be contained and controlled by formal operating parameters of the organization (Schmidt, 1997): strict procedures to be followed (as a script - a good practice), a rigid hierarchy and division of labor, function allocation, etc. This has been assumed to be the basis for safe operations in the human design processes in NPPs. Thus, reducing the variations in human response and individual autonomy, a potential source of human error and procedural violations, are an essential requirement for working in high-risk organizations. NPPs are no exception (La Porte and Thomas, 1995; De Terssac and Leplat, 1990; De Terssac, 1992; Hirshhorn, 1993; Vicente, 1999).

However, empirical ergonomic field study findings (Amalberti, 1992, 1996; De Terssac, 1992; Poyet, 1990) suggest that under actual working conditions, where there are performance standards to be met, and where skilled personnel are consistently using alternative strategies, what some people call human error or routine violations can be viewed as an innovative method used by these experts to cope with complex day-to-day situations. Under these circumstances routine violations can be understood to be part of the process for improving operating efficiency and even learning opportunities on the job; but too often they are seen as increasing the potential risk of accidents (Besnard and Greathead, 2003).

For Amalberti (1992) cognitive flexibility, and the local and contingent knowledge developed by the pilots may be an efficient and effective way to reduce and correct errors: pilots can recover from system errors; they adapt and they are capable of interpretation. The use of procedures have been studied by De Terssac (1992): "operators fill out the blanks and comply with the implied elements in the procedures." For Hatchuel (1994) operators can allow procedures to exist: "... the operator does the work in real time, which for those who developed the procedure is proof that the content and the time predicted for the execution of the task was correct; but the difficulties encountered in doing their activities require the operators to juggle competing activities in order to accomplish their tasks under the prescribed conditions." For Amalberti (1996) and Poyet (1990) removing the human element from direct control of production and limiting the possibilities for human intervention would be harmful, especially in situations where the system is disturbed, once humans develop strategies to deal with unexpected situations: “... operators are able to react and to update the prescriptions as a function of the context of the activity" (Poyet, 1990). Looking at routine office work Suchman (1987) said "that $\ldots$ action depends on local interaction between the actor and contingencies that, while they are accountable to a (given) plan or objective, remain essentially outside the scope of the plan", introducing a dichotomy between plan and action. Dekker (2003) believes that organizations must understand the reasons behind the gap between procedures and practices. From this perspective, errors must not always be interpreted as incompetence (Rizzo et al., 1995). Van der Schaaf (2000) goes further, and argues that rules in organizations are often developed simply to protect management from legal action.

Ergonomic field studies during the actual operation of NPPs are rare (and the publication of results more so still), probably because of the complex nature of a NPP and because of issues of safety and security. However, a set of field studies in Canadian nuclear power plants (Vicente et al., 1997) stresses the active problem-solving nature of monitoring activities, as the operators use proactive strategies to cope with the constraints imposed by the man-machine interface (e.g. changing prescribed variables such as set-points and alarms levels), indicating that actual monitoring activities can be quite different from the prescribed ones.

An examination of the ways in which control room operators deal with formal constructs and other organizational constraints while they are actually on duty will help to improve our understanding of cultural, organizational and cognitive issues and shed more light on fundamental (and still unresolved) issues in NPP operation such as: does following procedures (or not committing violations) always constitute the best practice? Do some violations of NPP operating procedures improve safety? What skills do operators need to optimize performance ?

\section{Research methodology}

Activity Analysis (Engenstrom, 2000), Distributed Cognition (Hutchins, 1994), and Cognitive Work Analysis (Vicente, 1999) provide the basis for our 
methodological framework. The subjects (NPP operators) were systematically observed in their actual work environment (control room and simulator). Our focus is on the description of the distributed regulation mechanisms (in the sense that operators work as a crew), both in nominal and degraded situations. This is a more tightly focused position than is normally used in traditional ergonomics, which usually applies few constraints on what is observed. The study observes how agents regulate (adapt) collectively their work when confronted with 3 types of situations: during an increase in workload (startup and shutdown of the plant), in situations where micro-incidents (MI) occur (Bressolle et al., 1996), and in dealing with critical accidents (in the simulator). We define a MI as any event that causes a disruption in normal operations that causes a divergence from the prescribed organization constructs (as we will see in Section 6). Audio and video recorders and field notes are used to collect empirical data on conversations and interaction that occur naturally within the work environment. Codification schemes are used to analyze the verbal protocols.

We want to emphasize that this method of field observation is especially suited to addressing the deeper cultural issues of the organization, by providing access to the backstage activities where workers keep the tacit competencies that make the cooperative strategies essential to the performance of daily activities possible. This strategy of gathering data allows vivid social scenes to be captured with all the conflicts, misunderstandings, the negotiations among actors, the creation of consensual arrangements to disregard the prescriptive rules that often come with the job, as well as site-specific jargon, gestures, jokes, etc.

\subsection{Participants}

Five control room operator crews from one NPP were selected to participate in this study under different operating conditions: a complete work shift (including the shift changes) in the plant during a planned shutdown; during the preparatory tests for startup; during the startup of the reactor and in simulator training. A control room operating crew is composed of 4 licensed operators - the Shift Supervisor, Foreman, Reactor Operator (RO) and the Secondary Circuit Operator (SCO) - and 1 unlicensed operator - the Auxiliary Panel Operator (PO). The Shift Supervisors and Foremen are Senior Reactor Operators ranging from 30 to 55 years of age and having more than 10 years experience in NPP operation (they had been operators in another NPP before coming to the plant used for the study). Some ROs and SCOs (aged 30-40 years), were also operators with 5-10 years experience having come from another NPP, but others ROs were recently hired workers ( 1.5 years) with ages from 20 to 25 years, and no previous experience in plant operation. They had received training during their time on the job.

\subsection{Procedure}

The procedure contained three phases: (1) data collection, (2) post-scenario debrief interviews, and (3) analysis.

\subsubsection{Data collection in the nuclear power plant}

For a better understanding of the language and jargon used as well as to gain insight into the NPP operating process, the study used analyst/observers with a prior background in nuclear energy. Authorization was obtained to have four analysts in the control room during the field observations, each one observing and collecting data from a licensed operator. Three fixed video cameras in the control room and micro-recorders in the pocket of each licensed control room operator were used to support data collection. The data collected consists of a total of $40 \mathrm{~h}$ of observation including reactor shutdown (12h), startup tests (6h), startup (6h) and simulator training $(16 \mathrm{~h})$ and involves a total of 5 operator crews.

\subsubsection{Post-scenario debrief interviews}

Debriefing interviews were carried out with the Shift Supervisors at the end of each observation period, where critical decisions and respective problem solving strategies made by the operators during their work activity were reviewed. In order to probe certain strategies more thoroughly, we asked questions about the cues that operators used to make situation assessments, the goals the operators had at particular points during the situation, whether they considered alternative courses of action when making a particular decision and whether the situation at hand reminded them of a previous experience. At the time of the observations, there were also informal interventions by the analysts to clarify some points, based on the questions used in the debriefing interviews. These conversations were transcribed along with the verbal communications of operators.

\subsubsection{Analysis}

The analysis was conducted following the criteria used for protocol analysis (Ericsson and Simon, 1993) and content analysis (Krippendorff, 1980): construction, recording, observation, and verbalization data from operations during a MI (verbalization, self-confrontation of the principal operators, debriefing interviews). All data were analyzed in terms of the collective interaction of the smaller sequences to derive the macro-sequences describing the crew's behavior.

The basic steps in the process are as follows: 
Table 1

Codification scheme

\begin{tabular}{|c|c|}
\hline Category & Definition \\
\hline Emergence & $\begin{array}{l}\text { The change in the nominal operation mode leading to a new course of action (COA), to do nothing, or to wait. } \\
\text { For example, to shut down the process or to wait to see how the incident escalated. }\end{array}$ \\
\hline Input & $\begin{array}{l}\text { Information leading to an altered assessment needing a solution. Identification of when the problem solving } \\
\text { related topic was introduced and what new factors caused the change. }\end{array}$ \\
\hline Instigated by & Who identified the need to tackle the problem. \\
\hline Involved & Team members involved from problem identification to the solution. \\
\hline Goal & The objective of the actions taken. Stated verbally or inferred by the researcher. \\
\hline Reason & $\begin{array}{l}\text { Based on the goal. For example, the goal may be to shutdown the process, the reason was to minimize } \\
\text { escalation potential. Could be stated but frequently had to be inferred. }\end{array}$ \\
\hline Options and consequences & $\begin{array}{l}\text { Options available as alternative means of resolving the problem identified. This could be not to do anything or } \\
\text { to wait. The consequences referred to what would happen if these were selected in contrast to the chosen COA. } \\
\text { Options and consequences could be stated but mostly had to be inferred. }\end{array}$ \\
\hline Time & The time taken from when the problem was identified until the decision was made. \\
\hline
\end{tabular}

(1) MI identification. The analyst's field notes listed events in chronological order. These notes were then organized during various working sessions to rank the MIs according to their importance for the study.

(2) The recordings of the operator conversations during the selected MIs were transcribed.

(3) A correlation between the MIs and the verbal protocols was made for each operator using coding criteria (see Table 1). The coding criteria were developed through systematic analysis of the study protocols and the objectives, and were used to identify the critical components related to a given cognitive strategy.

(4) Cognitive strategies were classified in terms of: (a) application of existing knowledge and action plans (rule-based), or (b) analytical problem solving including inductive and deductive reasoning, and imagery (knowledge-based), or according to the collaboration of other agents.

\section{Results: micro-incident description}

Under normal conditions, NPP operations are wellcoordinated and based on procedural instructions. In this "nominal" operating mode, the Foreman reads the procedural instructions aloud to the RO and SCO who then execute the instructions (see Fig. 1). When a MI occurs a more cooperative operating mode occurs. The Foreman stops reading the instructions and walks around the control room to be closer to the operators, monitor the instrument readings and so forth. Fig. 2 shows the Foreman's moves during the start of a reactor shutdown, when he reads the shutdown procedure from the pulpit. He was observed visiting the RO and SCO workstations nearly 40 times in $2 \mathrm{~h}$, showing the dynamics of the operation, even during a (simple) change of status in the plant.

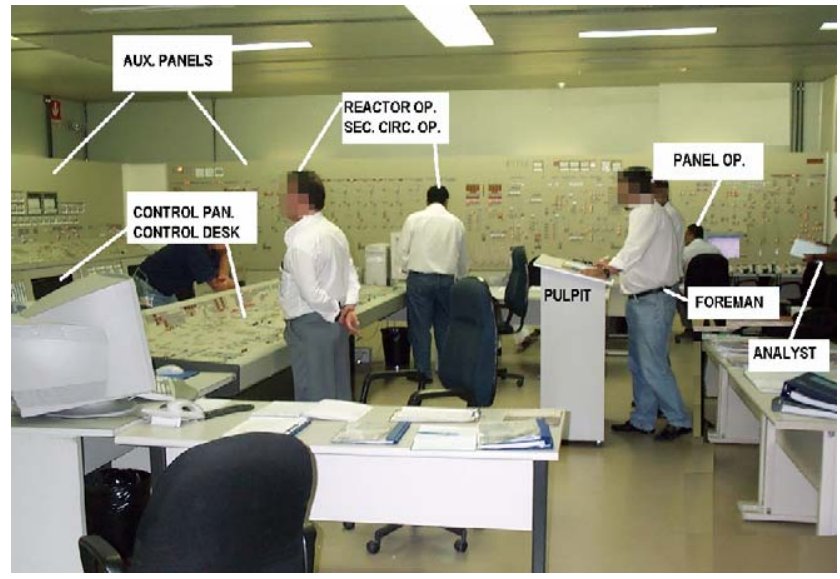

Fig. 1. Crew configuration during shutdown procedure-nominal operation mode.

The list the MIs studied is shown in Table 2. Selected MIs will be described and discussed in this section.

Boiler startup MI. The boiler is needed to provide steam for some functions after the reactor trip during the shutdown phase of a nuclear reactor, when the main heat source is lost. Since the plant uses the boiler only sporadically, it receives special attention (maintenance, tests) from the previous shift in preparation for operation. During the shift changeover, just before beginning the shutdown procedure, the operators access the boiler status.

Out-going Foreman: "Boiler 1, changing the probe." SCO:

Out-going Foreman: "It's done. It's being tested."

SCO:

"It is being tested. The operator will be done ..."

Note that the final information is not conclusive: "The operator will be done..." The information that the operators really need-whether the boiler can be 


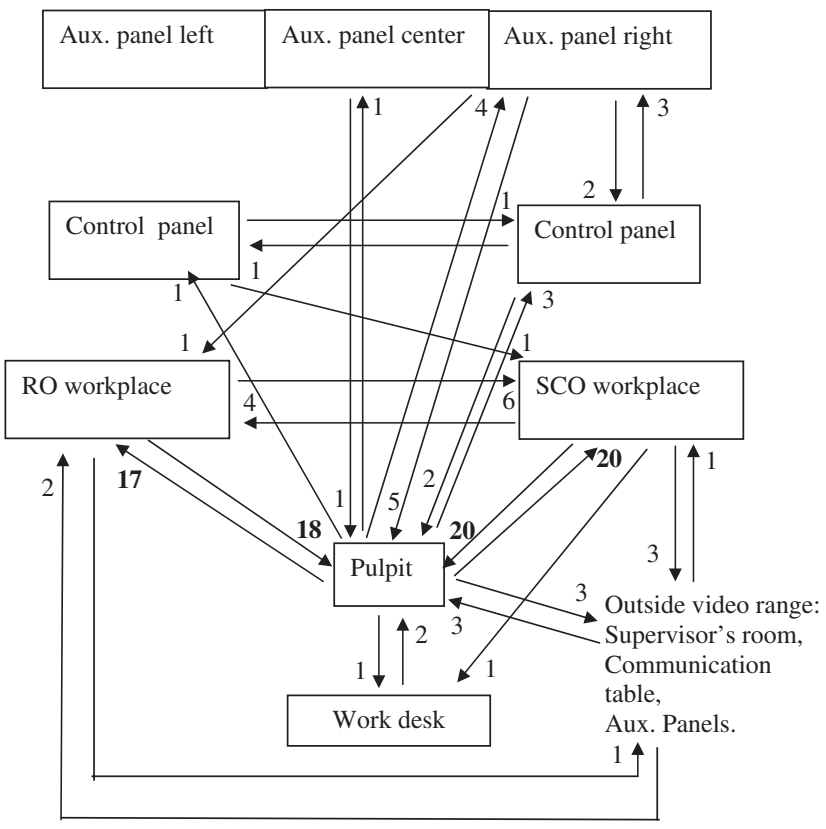

Fig. 2. Foreman movements during the start of the shutdown procedure from 21:13 and 23:01 o'clock. The control room is shown as a schematic divided according the position of the panels.

used - was not available at this time (about $3 \mathrm{~h}$ before connecting the boiler). Two hours later, another operator was observed attempting to get more conclusive information about the operational status of the boiler.

$\begin{array}{ll}\text { PO: } & \text { "Was Boiler } 1 \text { finished ..." } \\ \text { SCO: } & \text { "Yes." } \\ \text { Foreman: } & \text { "So . . theoretically it is available." } \\ \text { SCO: } & \text { "But is it still hot?" } \\ \text { PO: } & \text { "Ok! I'm going to start warming it up." } \\ \text { SCO: } & \text { "Because we will need the boiler soon, } \\ \text { PO: } & \text { "I'm going to the early morning." } \\ \end{array}$

According the first statement the boiler 1 is available. With the word "theoretically", the Foreman indicates his concern about the actual state of boiler 1 . When the SCO goes further, when asking if it is already warm, he committed the PO to an action: to heat the boiler 1 . Committed to action, the PO gave an unexpected answer: "I'm going to start boiler 2." The boiler 2 is the redundant boiler, which had not gone through testing on previous shift. From his answer, it can be inferred that PO had also some concerns about state of boiler 1. As will be shown in the MI analysis, the reason for the decision to start boiler 2 instead of boiler 1 was the presence of a scaffold near boiler 1: Since he knew about the scaffold from conversations with field operators, the PO was not sure about the real state of boiler 1.

As the time for connecting the boiler to the circuit approached, boiler 2 failed to start. At this point, the Supervisor asks the PO to go to the area and assess the situation of the boiler.

Supervisor: "Look at the condition of this f...ing
boiler! What is going on here?!
30 min later PO returns from boiler area.
PO:
$\begin{array}{ll}\text { "There is a scaffold down there that was } \\ \text { (interruption)" }\end{array}$
Supervisor: "Why should this keep the boiler from
PO:
Starting?!"

To assess the status of boiler 1, the operators use imprecise or vague communications constructs, common in every day conversations and used even in nuclear plant operations. It seems to be the only way the operators have to deal with system complexities and manage fresh information. Written documents, such as work permits, shift changeover registers, etc. are being filled in this very moment and cannot be used by the operators, especially in this case, where fieldwork was involved. Although the formal information (a closed work permit) indicates that boiler 1 is available (and it is!), the presence of the scaffold (a vague construct) triggers PO concerns about what the actual situation of the boiler 1 should be. At the last moment, he has to go to the area himself and talk to the field operators to clarify the situation.

Instrumentation test related MIs. These MIs were related to the execution of instrumentation tests during the shutdown. According to shutdown task procedures, instrumentation tests should be initiated just after the reactor trip at 23:30 o'clock. However, the test procedures say that the tests should be done only when the reactor has reached a sub-critical cold state. It takes a minimum of $6 \mathrm{~h}$ to get to a sub-critical cold state after the reactor trip. This situation provides a vivid example of two operations in conflict (the task planning and the test procedure). That creates problems for the operation. To resolve the conflict the Supervisor tried to understand the reasoning behind the sub-critical cold test requirement.

Instrument

Technician arriving in the control room: Supervisor:

Instrument

Technician:

Supervisor:
"Is it shut down? Can we proceed ...?" (with the tests)

"The reactor has been turned off. What do you need for the test?"

"It has to be in a sub-critical cold state."

"Well, it will not be cool until around 5 o'clock. Right now it's in hot sub-critical state." 
Table 2

Micro-incidents list

\begin{tabular}{|c|c|c|c|}
\hline Reactor shutdown-crew 1 & Pre startup tests - crew 2 & Increase reactor power-crew 3 & Simulator - crews 4 and 5 \\
\hline Boiler 1 startup & $\begin{array}{l}\text { Incompatibility between } \\
\text { procedure requirements }\end{array}$ & $\begin{array}{l}\text { Limitation system parameter } \\
\text { oscillation }\end{array}$ & Use of EOP procedures \\
\hline Instrumentation tests & Key operation on the control desk & Leakage in MKF tank & $\begin{array}{l}\text { Reduce pressure to } 80 \text { bar } \\
\text { according EOP }\end{array}$ \\
\hline $\begin{array}{l}\text { Reactor heat removal circuit } \\
\text { blockade after pump shutdown } \\
\text { Use of shutdown procedure }\end{array}$ & & & \\
\hline
\end{tabular}

Instrument

Technician:

Supervisor:

Five minutes later

the Instrument

Technician returned.

Supervisor:

Instrument

Technician:

Foreman:

Instrument

Technician:

Supervisor:
"Well, for this test ..."

"Now what we have to check is: how this test effects the primary circuit... Take a look at the test... see what conditions are really necessary to meet these requirements..."

"Can we do it or not?"

"The test we're going to do is just the part on train 1 ... signals that are changed on the

Limitation System rack for train 1 ... For Limitation to work, we would need to have... 2 of 3 ..." (interruption)

" 2 of $4, \ldots$ but the reactor is already shut down! I don't care if the Limitation System is on... The reactor is shut down."

"The problem is that the test is ... I doubt that most of it has been invalidated."

"Ok, Lets go. Get out of the Control Room. I have a lot of things to do! I can't stay up with you all night!"

The authorization again came without conclusive information. Why is it necessary to do the test with the reactor in a cold sub-critical reactor state? To get valid results, or not to jeopardize the operation (with the residual heat removal (RHR) process still underway)? A limitation of procedures took place: operators are told what to do, but they are not told why things should be done this way. By not explaining the reasons for the requirements and checking the situation, or explaining other requirements (such as task planning), the operators are forced to use more complex cognitive strategies at the knowledge level (inductive or deductive reasoning, inferences, etc.) in order to construct explanations for the requirements. In other words, the very procedures used to simplify cognitive strategies can have the opposite effect. In moments of intense activity this can lead to simplifications in the decision making process (the actual test influence in the operation was not taken into consideration) to reduce the operators cognitive load. As a result, right after beginning of the tests the control room alarm sounds. The following dialog describes the situation.

Supervisor: "Wait a minute! What's going on here?" (referring to the alarms)

Foreman: "This business of starting the test ..., the alarms go off all the time, man."

Supervisor: "We are in trip risk, right!" (laughing)

Supervisor: "I want to tell you something. To be in here with this sound going off is awful! Lets turn the alarm in the rack off!?"

The only way to turn off the alarm is by going inside the alarm rack, a routine violation. However, this violation will clearly help the operation, since the reactor is already tripped and the alarms were a nuisance and disturbing the operation. However, this was not the only problem facing the operators at the time. The automated system uses the same signals used by the alarm system and spurious blockades in important systems, such as the Reactor Residual Heat Removal System, were in progress. The reactor operator said: "We don't know if the stoppages occurred because of the test or because of a real problem at the plant."

Two hours after the authorization (2 AM), the Supervisor, pressured by the other operators, decided to stop the tests. Two hours later (4 AM), when the Reactor Heat Removal was in operation, the Supervisor tried the tests again but the same problems came up and at 5:15 AM, the Supervisor stopped the tests for the second time.

Reactor heat removal circuit block after pump shutdown MI. According to reactor shutdown procedure, 2 of the 4 reactor refrigeration pumps must be turned off 
(in this phase they were the main heat source). Immediately after the operator turned off the pumps, 1 of the 3 heat removal circuits in operation was unexpectedly blocked by the automatic system, initiating the MI. The operators realized that the halt was due to the overlap in shutdown curve pressure limits. For some unknown reason for the operators, the pump shutdown caused a slight increase in the pressure (33-36 bar), but enough to trigger the automatic system (the set point is in 34 bar). This occurred at the end of the shift. Operators from two different crews discussed the problem and how to restart the cooling circuits. They discussed two options: opening the breaker to physically bypass the interlock, or lowering the primary circuit pressure using the spray system.

Arriving RO:

Arriving Foreman:

Arriving RO:

Leaving Foreman:

Leaving Foreman:

Leaving RO:

Arriving Foreman:

Leaving RO:

Arriving Foreman:

Leaving Foreman:

Leaving Foreman:

Arriving Supervisor: "...last time what we did was lower the primary pressure here."

"I told him I would do this, but he (the Arriving Shift

Supervisor) is not sure about it."

"It can be lowered manually! Look, I know a trick that you can use to get control with lower pressure."

"You gonna cheat the big brother, aren't you?" (laughing) "We have done our job right.

You see, it is $69^{\circ}$ - to get to 50 by $11 \mathrm{AM}$, I think is reasonable..."

"But it's been some time ... and its not getting any cooler."

"Ask him to open up over there; the guy is in a hurry!"

"Tell them over there to open the breaker."

"Wait! We're not getting a reading ... the pressure is still too high!

"I think it's high, too. We'll have to drop the pressure a little. I will talk to (Supervisor name)."

"The (RO name) says that this problem has happened before with him. He also said that they lowered the pressure manually $\ldots$ in the core. We switch to manual, spray and later we go back... it's just to bypass the block... and to come back with the JN (heat removal circuit)." "Ok. Let's go."
After activating the pressurizer spray the primary circuit pressure dropped, and the heat removal circuit returned to operation. The shutdown process is completed one hour later, about $7 \mathrm{AM}$, when the reactor reached the sub-critical cold state (coolant temperature $50{ }^{\circ} \mathrm{C}$ and no pressure).

This MI exemplifies some of the difficulties caused by the design of the automation system (a hardware constraint) and the shutdown procedure (a formal construct) for the operators. The unexpected block forces the operators into a complicated diagnosis process to restore the refrigeration capability of the heat removal system. Based on their mental models and situation awareness of what should happen next (Endsley, 1997) - they discussed ways to bypass the interlock system - routine violations according to Reason's (1990) definition, or ways to improve performance in restoring the refrigeration system. The two main proposals were to disconnect directly from the circuit breaker, or a more subtle one-the one that was chosen - to use the spray system to lower the primary pressure by about 2 bars, thus staying within the limits of shutdown curve set point.

This MI also illustrates that the knowledge acquired by individual teams in unexpected situations is not necessarily shared with the rest of the organization, unless it occurs between operator crews and during repetition. This suggests some operational questions: Is the shutdown curve set point too tight? Is there some problem with the accuracy of pressure transmitters? Is there really a need for this interlock at this very moment? If not, in what situations should it be important (since it forces operators to circumvent the automation system)? The problem here is that such questions are dealing with the design of the automation system and the philosophy of the reactor control system, and whose answers lie elsewhere in the sociotechnical system (the NPP vendor, engineering department, regulator), but certainly outside the scope of the operators. This means that the operators appear to have a non-explicit assignment to deal with such situations as best they can. To respond to these implicit organizational demands the operators construct implicit operational measures that are not formally discussed; they only come up when the situation is repeated-"I have seen this situation before ..."

The next MIs occurred during the plant pre-startup tests with a different operating crew.

Incompatibility between procedure requirements $M I$. The operators follow the procedures for executing the primary circuit leakage test, prior to reactor startup. According to the procedure, the operator must electrically disconnect the pump during the test. However, with the pump turned off and the output valve closed and locked, is it necessary to electrically disconnect the pump? The problem here is if the operators disconnect 
the pump electrically, they also have to do the pump test and that takes about $8 \mathrm{~h}$. This same test had been performed the day before and was not scheduled to be done again in the task plan. This situation once again raises questions about the meaning behind procedures requirements, and with a different operating crew. During the discussions with the operator crew, the following dialog summarizes one of the fundamental questions about procedural constructs and their temporality.

Foreman: "What is the relationship between the Operation Manual, blocking this thing and the test (pause)? We are doing this test in the same region of the Operation Manual. (If)... you are here, at this point, then (the procedure writer) knows that the valve has already blocked it. Theoretically, the guy who wrote the procedure ... knows the plant condition. Then it would be redundant with what is written here. I am wondering if this double block is really necessary or if there is something else involved ... to block both the pump and the valve. What is (the procedure writer's) primary concern?"

RO: "He is not asking for the valve, in here, no."

Foreman: "I know. The valve he is talking about is here ... in the Operation Manual. He says that this valve has to be closed during the test. Because like he says: the valve has to be open after the test. During the test, it has to be closed. Then, why, if it is already closed there, did the guy insist that the valve be electrically disconnected!?"

When the Foreman said: "... the guy who wrote the procedure ... he knows the plant condition," he means that whoever prepared the test procedures should have specified the tests that need to be done for reactor startup in order, according to the status of the plant and the results of previous tests (it makes no sense to test the pumps twice). Operators faced incompatibility in timing in the three documents: the Operation Manual, the test procedures and task planning. Each one was prepared by a different group of specialists, in different organizations, at different points in time - in completely different contexts; nevertheless, they are supposed to be followed without the need for human intervention (interpretation) and they must be consistent with the rationale of the operation. At the end of about a 20 min discussion, and after checking the engineering/instrumentation diagrams, the operators decide not to disconnect the pump electrically during the test, ignoring the test procedure requirements and using the mutual situation awareness they achieved as a reference.

During a reactor power increase procedure, with a different (third) operator crew, the following MI occurred.

Limitation system parameter oscillation MI. To solve a problem of oscillation in the limitation system parameter in low power $(12.5 \%)$, the RO, after first consulting by phone with instrumentation technicians and with the Supervisor, increased reactor power by $5 \%$, to see if the oscillation stopped. This dialog begins when the RO, after halting the power increase procedure, explained the oscillation to the Supervisor.

RO:

"It is oscillating, man! ... The problem has started... look! ... from 12.5 it went to 28 ! When it was in 12.5 it should have been changed to 17.5 and it didn't move... it was stuck! And you could see that only it if you were passing by. From that point, it went to 28 , and... Now it is oscillating around that. Look!"

Supervisor: "Did it come back? It came back to 20."

RO on the phone: "Do you think we can increase the power a little bit to avoid the oscillation?(pause). The flow is low, real low! No, it isn't normal! The flow has to rise by more than $10 \%$, otherwise we will not get out of the oscillation point.(pause) Ok, but to increase the flow, we have to increase the power! (pause) Ok, by how much, more or less? Ok, bye."

RO:

"(Supervisor name), he suggested increasing the power by $5 \%$, to see if the feedwater flow increases by enough to get out of the low zone."

Supervisor: "Ok. Increase the power."

These conversations illustrate the importance of the operators' mental model and situation awareness to the installation's performance and safety. When the RO noticed the oscillation around the limitation system parameter, he immediately put the power increase on hold, reported the problem to Supervisor and asked for the help of the Instrument Technician. The oscillating parameter is made up of many signals. One of these is the feedwater flow, which was very low. The instrumentist recognized the pattern in which small variations in a 
low flow can give spurious signals, inferring that this might be the cause of the oscillation. The low flow could only be increased by an increase in the reactor powerbut the increase in the reactor power was stopped because of the oscillation problem - creating two conflicting conditions. The decision to increase the power by $5 \%$ (another violation of routine, since no formal construct considers the possibility of raising a $1300 \mathrm{MWE}$ reactor by $5 \%$ just to see if the oscillation stops) was successfully initiated by the operator crew.

Two other operator crews were observed while dealing with postulated accidents during the full scope simulator training. The next MI occurred in this situation.

Reduce pressure according to EOP MI. In dealing with an identified accident, the operators use the Emergency Operating Procedures-EOPs, where each item corresponds to an action to be carried out. It is presumed that after following all steps in the Procedures the consequences of the accident will be mitigated.

The MI occurred when the operators, following the EOP, set the primary circuit pressure to 80 bars. The instructor interrupted the training section and went to the control room. He told the operators that they should look at $P \times T$ graphic (on the computer screen) before reducing the pressure to $80 \mathrm{bars}$, even if the procedure says to do so unconditionally. The operators understand the problems related to the saturation of primary circuit promptly, but reply with the following statement: “... if we do not follow the procedures we are just $\mathrm{f}$..."

Following the instructor's rationale, in order to operate correctly, operators have to interpret the written procedure, correct and complement it to make the procedure an effective guide for action. Therefore, dealing with an actual accident could be a very complicated situation. As the operators stressed, they are trained to follow the procedures and are held accountable if they do not. According to the classical safety engineer paradigm, operational instructions are the end result of the process of the capitalization of knowledge and experience and must be always correct. In clear contraction with the reality of the operation, this situation demands a construction of a procedurereading-interpret habits, which appears to be broadly underestimated by the formal organization.

The theoretical plans and procedures or instructions are just some of the resources available for carrying out actions. The fundamental point to be considered is not so much the problem of the plan or of the procedure or instruction, but rather the idea that action/cognition calls on other resources, i.e. the material, social, and cultural characteristics of the environment in which events occur and which constitute the situation of the agent(s). As these characteristics can change at any time, to be adapted to them, individuals adjust their actions to the new environmental circumstances. If this is not supported by formal constructs, the adjustment is done on an ad hoc basis.

\section{Discussion}

The discussion focuses on the cultural and cognitive issues in NPP operation: control of MIs by operators; mental models, violations and safety; formal constructs and ad hoc configurations; and roles of the shift supervisor.

\subsection{Operators' activities while controlling micro- incidents}

When the operator becomes aware of a MI, his attention turns to information related to the MI, which he acquires by reading signals (as opposed to simply identifying the presence or absence or threshold overruns, which are a matter of indices or signals). Gathering and analyzing the information related to the MI becomes the focus of the operators attention. There is the moment when the agent concentrates on a single event, when he quickly excludes other information from his field of consciousness, and when he sets his mind to taking the meaning of the information he is givensomething he can do only if he concentrates. It is reasonable to assume that there is a strong relationship between reading activity - reading instruments, displays, etc. - as well as verbal and phoned communications and a temporary mind-set in which the field of attention is focused on a single event or series of events, temporarily inhibiting and excluding everything else. It is precisely this sort of activity that dominates MI operation activity. This reading activity and the search for meaning constitute a cognitive closed loop that enable the continuous generation of problem solving strategies. These strategies are mainly based on condition-action rules that are created on an ad hoc basis, supported by operators' tacit knowledge, mental model and underlying assumptions. In this moment, as the collaborative strategies emerge, operators move to a different location in the control room, talk to each other, adjust controls, communicate with field operators, interpret instructions, fill checklists (if available) and wait. Fig. 2 shows the dynamic nature of the operation.

\subsubsection{Dealing with distractions}

It is important to keep in mind that there are constant changes in focus and mind-set during the evolution of the MI. Every moment, the agent has to discern precisely what he perceives, so he restricts his field of visual perception and attention focus. Such behavior can cause a sort of Tunnel Vision, that is the lack of a high level picture of the system's structure (Casey 1986). In most cases, the reading of signs needs leads both 
semantically and spatially to additional information, but also to move the agent physically to another point in the control room (see Fig. 2), and to another kind of reading - as it occurs when the agent reads a value off in a display — or it can take him to a document (procedure, P\&I diagrams, logical drawings, alarm list, etc.), which must be pulled from its classification system and thumbed through until the right sheet is found. There is then a new focus of attention, but the operator must maintain his awareness all the time needed to cope with the MI. These constant changes in focus keeping the situation awareness must be managed by the agents' working memory.

Additional interruptions, or distractions, can cause them to lose their train of thought. The interference can result from changes in activity (and therefore of focus): while the agent is proceeding with an adjustment, something extrinsic to that activity interrupts him and requires him to suspend what he is doing and turn to something else. At a local level, these interruptions can be changes in focus: while the agent is reading off a series of values on a screen, the phone rings, or another agent needs an answer to a question; the agent responds quickly to the request and immediately returns to his task. We observed that these distractions are linked to cultural organization issues. For example, between 21:00 and 23:00 h, during the Boiler MI and the beginning of the Reactor Shutdown Procedure, the Reactor Operator answered 30 phone calls. Approximately $50 \%$ of the calls were related to the operational status of the plant (was it already down, primary temperature, etc.). Another $40 \%$ had nothing to do with operation (people asking for other people, copy requests and so on); and $10 \%$ of the calls related to the operator's needs.

This provides a clear example on how issues of organizational culture can interfere with the operators activity: people use the Control Room as a kind of Plant Call Center, since people know that the control room operators are always in their workplace and aware about plant status. Confronted with this number of phone calls one operator said, "It is really amazing ... at home, sometimes, I answer the phone: Control Room." It should be clear to the workers that in performing certain activities requiring closer attention, the simple fact of being interrupted (by a phone call, for example) could seriously affect the operator's state of concentration and commitment to the activity in progress. These interruptions are potential source of errors when agents return to an activity and try to pick up where they left off (Woods, 1984, 1995). For instance, if a phone rings when the reactor operator is filling out a checklist from an instrument readout he normally interrupts this work in progress, i.e. without completing it and mentally bookmarking his instructions. When he returns to his work, he might pick up at the wrong place on the checklist. Interruptions such as this require agents to perform additional marking and verification tasks in order to ensure the continuity of their activity. For example, during the shutdown procedure, the Foreman read the procedure and asked for information, while the reactor operator was reading values from graphic registers in another panel. The operator does not reply immediately: first, he finishes his readings, then goes back to the procedure and finds that he has to go to a new page to answer the questions; he turns to that page and only then turns to the Foreman to reply. He did not take the risk of interrupting the continuity of application of the instructions before reaching a stable and easily identifiable point. It is a characteristic of cooperative work; one agent can evaluate the priority of the call from another agent using different signals such tone and voice level, posture, attitude etc. Conversely, agents whose activity requires them to interrupt the activity of another agent develop an additional activity of following the other agents' activity and controlling the interruptions they have to provoke.

\subsection{Mental models, violations and safety}

In almost all MI studied the image that operators have of the system's behavior (their mental models) and what will happen in the near future (situation awareness) played a significant role in the success or failure of the selected course of action. For instance, in the instrumentation test MI, the crew was unable to obtain an accurate picture about the relation between the tests and the automation system. Even if there were no safety problem (they were aware of this since they knew that the reactor had been turned off), alarms and blockades jeopardized the operation. They committed a violation by turning off the alarm buzzer to make the operation easier under the circumstances-with the nuisance alarms and the reactor turned off.

In the limitation system parameter oscillation and the reactor heat removal circuit blockade after pump shutdown MIs an adequate but hardly constructed (through the interaction of many workers), situation awareness was achieved. The actions selected, both taken to bypass the automation system, proved to be successful. In the simulator MI, the instructor advised the operators to not go forward with the procedure instructions, without first getting an accurate picture of the $P \times V$ condition of the primary circuit.

It is, therefore, of paramount importance for the safety and efficiency of the operation that operators have an accurate picture of the system's behavior, and one that actually reflects what is happening. Violations only become a safety problem when they occur with inadequate awareness of the situation, as already pointed out by Endsley (1997). In this study, some problems related to human/automation interaction were found that can be specifically traced to systems design, 
since the decentralized distributed automation system does not provide an accurate and workable picture of plant behavior. Despite the fact that the plant began operations in 2000, most of instrumentation and control equipment had been acquired 20 years before (due to delays in the plant construction) and did not enjoy the benefits of recent technological evolutions in human/ machine interfaces from ergonomic research.

\subsection{Formal constructs and ad hoc configurations}

The results of this study confirm the ergonomics research findings (see Section 3) in which all susceptible events encountered by the actors in complex systems cannot be anticipated in formal organizational constructs, even in the highly regulated (and controlled) nuclear energy industry. There are parts of the reality of daily activities that do not fit into the model of explicit rules.

In this study, when operators were observed dealing with detailed procedures, a highly automated and decentralized control system with a number independent interlocks to protect equipment and systems, combined with inadequate information about the status of the automation system, were required to make many ad hoc reconfigurations of the system just to enable the system to function. For instance, in the RHR system block MI, a slight increase in the primary circuit pressure ( 3 bars) was enough to block one RHR circuit. To ensure the proper operation of the RHR system the operators had to make a contingent ad hoc modification of the automation system - to spray the reactor core to bring the pressure to within shutdown curve limits. Similar modifications were needed to turn off the alarm buzzer, or to increase the reactor power because of oscillations around the limitation system parameter. Indeed, these reconfigurations are object of humorous remarks: “... are you gonna cheat the big brother..." suggesting that these are common occurrences during the operation.

In other MIs, as in the incompatibility between the test authorization and procedures, the need for ad hoc reconfigurations result from procedural conflicts. Paradoxically, in these $2 \mathrm{MIs}$, the procedures created to minimize the need for human intervention, require this intervention; since human intervention is the only way to overcome, by local, contingent and tacit knowledge, the conditions that led to the constraints and to modify the procedures in the actual execution context for effective action.

Based on these findings, the study suggests that these actions should not be interpreted as violationsaccording to Reason's definition. Instead, they should be seen as ad hoc reconfigurations of the formal constructs (including hardware and rules) in situations that were not anticipated in the system design. They emerge as conditioned responses created by experts to cope with an unexpected situation and responding to an express need for additional or different working practices or tools. Indeed, in work situations where there are performance standards, such as in NPP operations, and where highly trained personnel show a consistent use of strategies (with good situation awareness), operators are able to perform safe ad hoc interventions. As shown in this study, flexibility in formal constructs, as a result of human interventions, enables a safe and efficient operation.

In that sense, the still vivid paradigm of high-risk organizations, in which the design of an exhaustive set of rules and procedures enhances system reliability, may actually result in opposite outcomes. One step towards a solution is to design rules and procedures in which operators understand the rationale and to which they will conform - as we saw in many MIs, the search for meaning in the procedure requirements was at the center of the discussions.

The problem here (especially for the operators) is the fact that we are not prepared to accept violations (ad hoc reconfiguration) if an accident occurs. The hindsight bias (Woods and Cook, 2002) of accident investigators, combined with a causal, event-driven accident model that covers the search for causes of accidents throughout the system - even when a perfect causal chain does not exist (Hollnagel, 2002), will hold responsible all those determined to have committed a violation (even when the very same violation occurred many times and with success). The situation creates anxiety in operators, and some of the short cuts, practices and tricks that differ from prescribed procedures are not formally discussed in the organization. They are shared only when the same situation is repeated: "... I have seen this situation before ...", as seen in several of the MIs. Operator anxiety was also shown in the discussions with the training Instructor: " ... if we don't follow the procedures we are just $f . . . "$

\subsection{The multiple roles of the Shift Supervisor}

According to the organization chart, the Shift Supervisors have the ultimate responsibility for control room operation and safety. They also serve as the main communications channel from the control room to the other parts of the plant, and even outside the plant. Because of these assigned tasks, the Supervisor plays multiple roles, both technical and administrative. He must follow procedures for checking the actions of the ROs and SCOs and consider the process beyond the instructions to give final authorization for the implementation of ad hoc reconfigurations, as seen in almost all MIs. Together with the Foreman, the Supervisor helps the operators organize access to resources (binders, operations sheets and logs, for example), and give instructions to panel operators, maintenance, 
instrumentation/test and engineering employees (see for instance the boiler and tests MIs). He must also coordinate contingency meetings to collectively solve problems caused by the MIs, exchanging information and diagnoses/prognoses of the dynamics of the system, critique the decisions of others and act as a clearing house for critical comments: "This thing ... test liberation, it sets off alarms all the time, man." Knowing the operators were right, he used his sense of humor to ease the tension: "We are all in trip risk!", when the reactor had been tripped. He does this not just when asked, but most of the time spontaneously. It is precisely in the case of spontaneous assistance that the operators best display their (ad hoc) innovations concerning their activity. See for instance, the reactor heat removal block MI, when the RO says: "... I know a trick where you can get more control with lower pressure."

During shutdown the Supervisor workload increases enormously, since besides the tasks described above he also must sign and authorize all work permits relating to the operation. As we saw, right after the reactor tripped, a number of employees came to the control room with their work permits asking for Supervisor authorization, as in the case of the instrumentation tests: "Is it ... shut down? Can we proceed?"

Plant managers, especially in the current environment in which NPPs are operating, impose other constraints on Supervisor activity. Primarily conceived as part of a government strategy, NPPs receive their budget from the states, no matter how much energy they produce. At the present time they have to sell the energy to the market at competitive prices in order to survive. The Supervisor acts as link between managers and operators, workers with diverse cultures and assumptions. In the debrief interview after the Tests MI, the Supervisor shed some light to the situation: ", I should not have authorized those tests, ok. I should not have authorized them! But people tried to do the tests, even though they have be done when the plant is in subcritical cold state. This is one of the requirements in the test procedure. Still they tried to do the tests before the sub-critical cold state had been reached. Because we have a very short down period! So I accepted the challenge and authorized the tests. But in the middle of the (...test...) I felt it was impossible and I ordered it stopped."

\section{Conclusion}

Our research develops an applied ergonomics methodology based on field studies that permit a more thorough knowledge of the NPP operator's cultural and cognitive issues. In this study, using practical examples, we show how cognitive strategies that emerged under environment constraints (incompatible procedures, interfering activities, pressures to do tasks, difficulties regarding $\mathrm{I} / \mathrm{C}$ and automation systems) can modify the culture (the way they do things) of the control room operators.

In this kind of ethnographic research, the main objective is rarely to hypothesize and test for statistical significance to add to generalized principles of human behavior, or to make general recommendations for organizational changes. Rather, this kind of applied research tends to emphasize the interactions and constraints on the natural (local) environment and that which is imperative within that environment to the individual performing a particular task, with possible implications for other organizational levels.

Even within the research limitations implied in modeling the activities of a larger number of operator crews, there is evidence to show that the safety implications of NPP operators cultural and cognitive issues go well beyond the normative work behavior prescribed by the organization formal constructs. The normative framework usually used in high-risk organizations to develop standards of competence and training programs, to assess human reliability, and even extending to accident investigation methods, and to blame people when violations occur, should be revised since it differs from what is required for effective and safe performance.

Our findings show that the competence required for the operators to improve their performance when dealing with MIs within organizational constraints (hardware, software, liveware) are mainly concerned with:

1. developing the possibility of constructing situation awareness during the course of action, understanding the task at a sufficiently global level to understand the most important demands set by the ongoing situation. To do so, operators must understand the procedures (especially the intent of the requirements) and the (possible) different ways to carry out their instructions, managing attention resources for doing that, but also building mental simulations concerning the future of the process, for his own procedures and those of the other operators' procedures (mutual situation awareness);

2. managing conflicts, gaps and time problems created by this procedure (ongoing task procedures), the plant's technical specifications, the task planning schedule and the demands from his colleagues, adjusting the different time constraints of the procedure, the other operators' procedures, and the process;

3. dealing with distractions, developing skills for collaborative efforts with the other operators and plant staff by accepting, postponing or rejecting the interruptions they bring to his own activities, and conversely by discovering the right moment to interrupt the others' activities. 
The cultural issues involved also concern the sharing of these competencies with the plant staff, since maintenance, instrumentation, engineering, and management personnel are in close contact with the operators. By understanding local patterns of interaction, or employees action behaviors, it should be possible to develop more workable environments, in which undesirable interaction patterns will also likely change. Thus, cultural issues can also have an impact upon design issues like those of preparing instructions and support activities, developing organizational roles, and designing training systems. For the plant we studied, our field work has made it possible to generate more specific design information. For example:

to develop an operator support system that provides more information about the status of the plant and automation system; to do more extensive work on procedures - the first objective will be to translate procedures into the operator's native language;

to use a senior operator to help the Supervisor deal with work authorizations during scheduled shutdowns;

to hire and train more operators to avoid $12 \mathrm{~h}$ shifts;

to distribute basic information about reactor status around the plant;

to study ways to improve the control room communications to the external areas of the plant.

\section{References}

Amalberti, R., 1992. Safety in process control: an operator centred point of view. Reliab. Eng. Syst. Saf. (38), 1-3.

Amalberti, R., 1996. La Conduite de Systèmes à Risques. Le travail Humaine, Presses Universitaires de France - puf, Paris.

Besnard, D., Greathead, D., 2003. A cognitive approach to safe violations. Cognition Techol. Work 5, 272-282.

Bressolle, M., et al., 1996. Traitement Cognitif et Organisationnel des Micro-incidents Dans le Domaine du Contrôle Aérien: Analyse des Boucles de Régulation Formelles et Informelles. Toulouse, France, Octarès.

Carvalho, P., Vidal, M., 2001. A Sociotechnical Review of the REDUC's Oil Pipeline Accident Occurred in 18-01-2000 in Rio de Janeiro. In: Proceedings of the International Conference on Computer-Aided Ergonomics and Safety, (CAES 2001), Maui, Hawaii, USA, Aug.

Carvalho, P., Vidal, M., 2003. Ergonomics and reliability of high risk organizations: the nuclear power plant operation under the complexity theory paradigm. In: XVth Triennial Congress of the International Ergonomics Association, (IEA 2003), Seoul, Republic of Korea, Aug.

Casey, B., 1986. A Games Approach to System Interface Design. IEEE Transactions on Professional Communications 29 (4), 67-71.
De Terssac, G., 1992. Autonomie dans le Travail. Presses Universitaires de France, puf, Paris.

De Terssac, G., Leplat, J., 1990. La Fiabilité et L'ergonomie: Spécificité et Complémentarité. Rev. Psychol. Appl. 40 (3), 377-386.

Dekker, S., 2003. Failure to adapt or adaptations that fail: contrasting models on procedures and safety. Appl. Ergon. 34, 133-238.

Endsley, M., 1997. The role of situation awareness in naturalistic decision making. In: Zsambok, C.E., Klein, G.A. (Eds.), Naturalistic Decision Making. Lawrence Erlbaum Associates, Mahwah, NJ.

Engenstrom, I., 2000. Activity theory as a framework for analyzing and redesigning work. Ergonomics 43 (7), 960-974.

Ericsson, K.A., Simon, H.A., 1993. Protocol Analysis: Verbal Reports as Data. Cambridge University Press, Cambridge.

Hatchuel, A., 1994, Apprentissages collectifs et activités de conception. Revue Française de Gestion, Juin, Juillet, Août, pp. 109-120.

Hirshhorn, L., 1993. Hierarchy versus bureaucracy: the case of a nuclear reactor. In: Roberts, K. (Ed.), New Challenges to Understand Organizations. Macmillan Publishing Co, New Work, NJ.

Hollnagel, E., 2002, Understanding accidents: from root causes to performance variability. In: Proceedings of IEEE 7th Human Factors Meeting, Scottsdale, Arizona.

Hutchins, E., 1994. Cognition in the wild. MIT Press, Cambridge, MA.

Krippendorff, K., 1980. Content Analysis. An Introduction to its Methodology. SAGE Publications Ltd, London, UK.

La Porte, T., Thomas, C., 1995. Regulatory Compliance and the Ethos of Quality Enhancement: Surprises in Nuclear Power Plant Operations. J. Public Admin. Res. Theory (5), 109-137.

Mosey, D., 1990. Reactor Accidents: Nuclear Safety and the Role of Institutional Failure. Nuclear Engineering International Special Publication, Quadrant House, Surrey, UK.

Poyet, C., 1990. L'homme agent de fiabilité dans les systemes informatisés. In: De, Tersac., Leplat (Eds.), Le Fateurs Humains de la Fiabilité. Toulouse, Octares.

Reason, J., 1990. Human Error. Cambridge University Press, Cambridge, England.

Rizzo, A., Ferrante, D., Bagnara, S., 1995. Handling human error. In: Hoc, J.M., Cacciabue, P., Hollnagel, E. (Eds.), Expertise and Technology: Cognition and Human-Computer Interaction. Lawrence Erlbaum, Hillsdale, NJ.

Schmidt, K., 1997. Of maps and scripts: the status of formal constructs in cooperative work. In: Proceedings of ACM Conference on Supporting Group Work, Phoenix, Arizona, pp. 138-147.

Suchman, L., 1987. Plans and Situated Actions: the Problem of Human-Machine Communication. Cambridge University Press, Cambridge, MA.

Van der Schaaf, T., 2000. Near miss reporting changes the safety culture. www.engr.wisc.edu/centers/chpra/newsletter/CHPCS vol5.1.pdf

Vicente, K., 1999. Cognitive Work Analysis. Lawrence Erlbaum Associates, London, UK.

Vicente, K., Mumaw, R., Roth, E., 1997. Cognitive Functioning of Control Room Operators-Final Phase. AECB 96-175, Atomic Energy Canadian Bureau, Ottawa, Ontario, Canada.

Wilson, J., 2000. Fundamentals of Ergonomics in Theory and Practice. Appl. Ergon. 31, 557-567 (Elsevier).

Woods, D., 1984. Visual Momentum: A concept to improve cognitive coupling of person and computer. Int. J. Man-Machine Stud. (21), 229-244.

Woods, D., 1995. The alarm problem and direct attention in dynamic fault management. Ergonomics 38 (11), 2371-2393.

Woods, D., Cook, R., 2002. Nine Steps to Move Forward from Error. Cognition Technol. Work 4, 137-144. 